\title{
Heterogeneous Stress Distribution and Hierarchical Structure in the Highly-Oriented Nylon 6 Strings Annealed at the Various Temperatures to Evaluate the True Crystallite Modulus
}

\author{
Sreenivas Kummara and Kohji Tashiro* \\ Department of Future Industry-oriented Basic Science and Materials, \\ Toyota Technological Institute, Tempaku, Nagoya 46808511, Japan
}

- Supporting Information -

\section{The thermal data of the highly-oriented nylon 6 samples annealed at the various temperatures}

Figure S-1 shows the DSC thermograms measured in the heating process of the nylon 6 string samples annealed at the various temperatures. The heating rate was $10^{\circ} \mathrm{C} / \mathrm{min}$.

As well known, a small subpeak is generally observed when a polymer sample is annealed at a certain temperature $\left(T_{\text {an }}\right)$, which corresponds to the melting of the small crystallites created in the annealing at $T_{\text {an. }}$. This type of subpeak is detected for the unoriented nylon 6 samples as reported in the literature. ${ }^{1,2}$ The half width of the X-ray diffraction peak might be sharper for the sample annealed at a higher $T_{\mathrm{an}}$, since the crystallite size becomes larger. However, this type of subpeak was difficult to detect for the present highly-oriented nylon 6 samples. Correspondingly, the crystallite size estimated from the X-ray diffraction peaks (200, 020 and 0014) were almost constant in the $T_{\text {an }}$ region below $180^{\circ} \mathrm{C}$ (see the text). For the samples annealed in the higher temperature region than $180^{\circ} \mathrm{C}$, the DSC peak positions did not shift but only the peak height changed remarkably. More concretely, the two peaks were detected at about 218 and $221{ }^{\circ} \mathrm{C}$ for the original sample. Even when the annealing temperature was increased in the region of $100-180^{\circ} \mathrm{C}$, these peaks did not change remarkably. Once the annealing temperature was increased higher, the first peak became stronger and shifted a little bit 
to the lower temperature side. This tendency became more remarkable, and the DSC thermogram of the sample annealed at $214^{\circ} \mathrm{C}$ showed a very sharp peak at $217^{\circ} \mathrm{C}$ attendant with the shoulder at $218^{\circ} \mathrm{C}$.

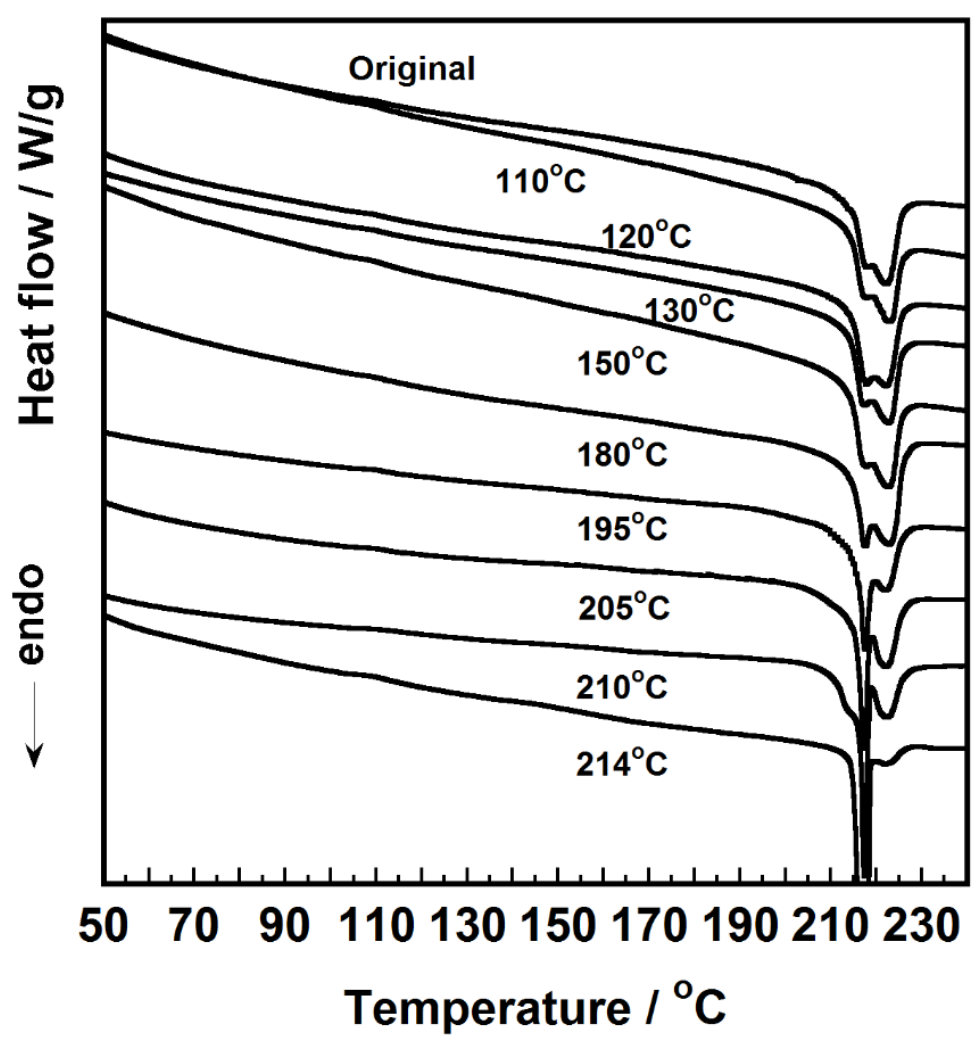

Figure S-1 DSC thermograms measured for the highly-oriented nylon string samples annealed at the various temperatures.

\section{References}

(1) Liu, T. X.; Liu, Z. H.; Ma, K. X.; Shen, L.; Zeng, K. Y.; He, C. B. Morphology, thermal and mechanical behavior of polyamide 6/layered-silicate nanocomposites, Comp. Sci. Tech. 2003, 63, $331-337$

(2) Millot, C.; Fillot, L.; Lame, O.; Sotta, P.; Seguela, R. Assessment of polyamide-6 crystallinity by DSC. J. Therm. Analy. Calorim. 2015, 122, 307-314. 